\title{
Enriching a cellulose hydrogel with a biologically active marine exopolysaccharide for cell-based cartilage engineering
}

\author{
Rederstorff E. ${ }^{1,2,3}$, Rethore G. ${ }^{1,2,4}$, Weiss P. ${ }^{1,2,4}$, Sourice S. ${ }^{1,2}$, Beck-Cormier S. ${ }^{1,2}$, Mathieu E. ${ }^{5}$,

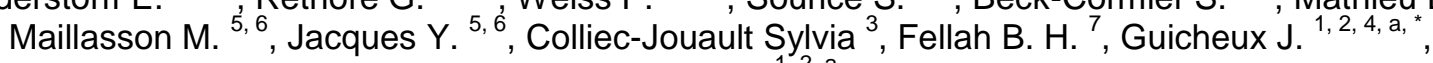 \\ Vinatier C. ${ }^{1,2, a}$
}

\footnotetext{
${ }^{1}$ INSERM; UMRS 791-LIOAD, Skeletal Tissue Engineering and Physiopathology (STEP) Group, UFR Odontology; Nantes France

${ }^{2}$ Université de Nantes; Unité de Formation et de Recherche (UFR) Odontologie; Nantes France

${ }^{3}$ French Research Institute for Exploitation of the Sea (IFREMER), Laboratory of Biotechnology and Marine Molecules; Nantes France

${ }^{4}$ Centre Hospitalier Universitaire Nantes, PHU4, Ostéo-articulaire Tête et Cou, Odontologie, Neurochirurgie, Neurotraumatologie (OTONN); Nantes France

${ }^{5}$ INSERM; UMRS 1087, L'Institut du Thorax; Nantes France

${ }^{6}$ Plateforme IMPACT Biogenouest; CRCNA-INSERM U892, SFR Santé François Bonamy/UMS INSERM; Nantes France

${ }^{7}$ Centre for Preclinical Research and Investigation of the ONIRIS; Nantes-Atlantic College of Veterinary Medicine, Food Science and Engineering (CRIP); Nantes France

* Corresponding author : J. Guicheux, email address : jerome.guicheux@inserm.fr

${ }^{a}$ These authors contributed equally to this study.
}

\begin{abstract}
:
The development of biologically and mechanically competent hydrogels is a prerequisite in cartilage engineering. We recently demonstrated that a marine exopolysaccharide, GY785, stimulates the in vitro chondrogenesis of adipose stromal cells. In the present study, we thus hypothesized that enriching our silated hydroxypropyl methylcellulose hydrogel (Si-HPMC) with GY785 might offer new prospects in the development of scaffolds for cartilage regeneration. The interaction properties of GY785 with growth factors was tested by surface plasmon resonance (SPR). The biocompatibility of Si-HPMC/GY785 towards rabbit articular chondrocytes (RACs) and its ability to maintain and recover a chondrocytic phenotype were then evaluated in vitro by MTS assay, cell counting and qRT-PCR. Finally, we evaluated the potential of Si-HPMC/GY785 associated with RACs to form cartilaginous tissue in vivo by transplantation into the subcutis of nude mice for 3 weeks. Our SPR data indicated that GY785 was able to physically interact with BMP-2 and TGF $\beta$. Our analyses also showed that three-dimensionally (3D)-cultured RACs into Si-HPMC/GY785 strongly expressed type II collagen (COL2) and aggrecan transcripts when compared to Si-HPMC alone. In addition, RACs also produced large amounts of extracellular matrix (ECM) containing glycosaminoglycans (GAG) and COL2. When dedifferentiated RACs were replaced in 3D in Si-HPMC/GY785, the expressions of COL2 and aggrecan transcripts were
\end{abstract}


recovered and that of type I collagen decreased. Immunohistological analyses of Si-HPMC/GY785 constructs transplanted into nude mice revealed the production of a cartilage-like extracellular matrix (ECM) containing high amounts of GAG and COL2. These results indicate that GY785-enriched SiHPMC appears to be a promising hydrogel for cartilage tissue engineering.

Keywords: cartilage, hydrogel, glycosaminoglycan, marine polysaccharides, chondrocytes, tissue engineering 


\section{Introduction}

Articular cartilage (AC) is an avascular connective tissue containing a unique cell type, the chondrocyte, which synthesizes an abundant and highly hydrated extracellular matrix (ECM) (Steinert, et al. 2007) mainly composed of type II collagen and aggrecan (Demoor, et al. 2014). AC unfortunately has a poor capacity for self-repair, and injuries to $\mathrm{AC}$ are thus irreversible and may lead to long-term joint degeneration (Gomoll and Minas 2014). Tissue engineering strategies involving the combination of cells, scaffolds and bioactive agents have emerged to build functional new tissue in order to replace damaged cartilage (van Osch, et al. 2009, Vinatier, et al. 2009). The ideal scaffold material for cartilage tissue engineering should be one which closely mimics the natural cartilage environment in a structural, mechanical and biofunctional way (Bonzani, et al. 2006, Griffith and Naughton 2002). Several studies have demonstrated that chondrocyte functions differ in 2D and 3D systems (Brodkin, et al. 2004, Mukaida, et al. 2005) with a progressive dedifferentiation of chondrocytes in $2 \mathrm{D}$ monolayer culture whereas this phenomenon is prevented by a 3D culture (Dehne, et al. 2010, Mukaida, et al. 2005). Given that articular cartilage is a tissue subject to considerable stress, to mimic the environments of cartilage tissue in structural terms, the fundamental structure of a scaffold must therefore be a 3D system. In this context, an injectable, self-setting silated hydroxypropyl methylcellulose (Si-HPMC) based hydrogel has been developed previously (X. Bourges, et al. 2002, Xavier Bourges, et al. 2002). This cellulose-based hydrogel once reticulated is composed of only $2 \%$ of dry polymer and $98 \%$ of water, composition that mimics the high hydration of articular cartilage ECM. In a previous work, this Si-HPMC hydrogel has been demonstrated as a suitable matrix for the 3D 
culture of chondrocytes in vitro (Vinatier, et al. 2005) and the production of cartilage in vivo (Vinatier, et al. 2007).

Biological sulfated polysaccharides such as sulfated glycosaminoglycans (GAGs) are major components of articular cartilage ECM. GAGs are implied in many biological processes like cytokinesis, cell proliferation, differentiation, migration, tissue morphogenesis, organogenesis and wound repair (Yamada and Sugahara 2008). GAGs can bind many different classes of proteins, ranging from growth factors (FGFs, VEGFs, PDGF, glial cell-derived neurotrophic factor (GDNF), and HGF), cytokines (interleukins, interferon, PF4, and RANTES), to metabolic enzymes (lipoprotein lipase) and structural proteins (laminin and fibronectin), among many others (Casu and Lindahl 2001). GAGs would control the diffusion of the growth factors to establish protein gradients or act as a repository of growth factors that could be sequestered from cells and released at an appropriate time (Zcharia, et al. 2005). The actions of biologically active polysaccharides are largely dependent on their molecular structure, in particular their molecular size and varying size of repeating unit features, osidic residues, linkage, and sulfation degree.

Natural polysaccharides derived from marine prokaryotes offer a significant structural chimiodiversity with novel and striking biological properties (Guezennec 2002). In addition, natural GAG-mimetics can be chemically customized and produced in large amounts with relatively simple and reproducible processes, making them potentially suitable as bioactive agents for medical applications. Among the large number of prokaryotic species capable of producing GAG (Guezennec 2002), Alteromonas infernus has been shown to produce a branched high-molecular weight polysaccharide: 
GY785 $\left(\sim 10^{6} \mathrm{~g} / \mathrm{mol}\right)$. This exopolysaccharide (EPS) is unique with no known analog. Low-molecular weight (GY785 DR) and low-molecular weight oversulfated (GY785 DRS) derivatives of GY785 are described as "'heparin-like" compounds. These GY785 derivatives have been shown to exhibits some anticoagulant properties (Matou, et al. 2005). Interestingly, they also have been recently described to positively influence the chondrogenic differentiation of Adipose derived stromal cells (Merceron, et al. 2012). In order to develop a biologically and biomechanically competent hydrogel for cartilage tissue engineering, we propose to associate the branched high molecular weight polysaccharide GY785 (0.67\% (w/v)) with Si-HPMC scaffold.

The aim of the present work was thus to investigate whether the enrichment in GY785 EPS improves the potential of Si-HPMC hydrogel for engineering cartilage. In this attempt, we first assessed the growth factors-interacting properties of GY785, then the cytocompatibility of Si-HPMC/GY785 was assessed with regards to articular chondrocytes. Secondly, the potential of Si-HPMC/GY785 to support the maintenance of a chondrocytic phenotype in vitro and to produce cartilaginous matrix in subcutaneous pockets of nude mice were investigated. 


\section{Materials and Methods}

\subsection{Materials}

Hydroxypropyl methylcellulose (HPMC) $\mathrm{E}^{\mathrm{B}} \mathrm{M}^{\circledR}$ was purchased from ColorconDown chemical (Bougival, France). GY785 EPS was produced by Seadev-FermenSys SAS (Plouzané, France) and sterilized using ethylene oxide by IONISOS (Gien, France). Hyaluronidase, actinomycin-D, trypsin/EDTA, type II collagenase (290 units/mg), HEPES (4-(2-hydroxyethyl)-1-piperazineethanesulfonic) and isopropanol were purchased from Sigma-Aldrich (St-Louis, USA). Hank's Balanced sodium salt (HBSS), DMEM 4.5 G/1 glucose), penicillin/streptomycin, L-glutamine, Bone morphogenetic protein-2 (BMP-2), Trizol ${ }^{\circledR}$, DNase I, Taq DNA polymerase and reverse transcriptase superscript II were obtained from Invitrogen corporation (Paisley, UK). Methyl Tetrazolium Salt (MTS) was purchased from promega (USA). A live and dead viability/cytotoxicity kit assay was obtained from Invitrogen. Fetal calf serum (FCS) was obtained from Dominique Dutscher (Brumath, France). Cell culture plastics were purchased from Corning (VWR, France). New Zealand rabbits were obtained from Grimaud frères (Roussay, France). Monoclonal antibody against type II collagen was purchased from Oncogene (San Diego, United States). Monoclonal antibody against type I collagen was purchased from MP Biomedicals (Illkirch, France). TGF- $\beta 1$ was obtained from PeproTech Inc. (London, UK). DIG RNA labelling kit was acquired from Roche Diagnostics (Mannheim, Germany) All other chemicals were from standard laboratory suppliers and were of the highest purity available. 


\subsection{Surface plasmon resonance}

Experiments were carried out on a Biacore 3000 instrument (Biacore, Uppsala, Sweden). TGF- $\beta 1$ and BMP-2 were covalently immobilized to the dextran matrix of a CM5 sensor chip (Biacore) as recommended by the manufacturer at a flow rate of $5 \mu \mathrm{L} / \mathrm{min}$. Binding assays of GY785 $(0.03125,0.0625,0.125,0.25,0.50$ and $1 \mu \mathrm{g} / \mathrm{mL})$ were performed in $10 \mathrm{mM}$ HEPES buffer, $\mathrm{pH} 7.4$, containing $0.15 \mathrm{M} \mathrm{NaCl}$ and $0.005 \%$ P2O surfactant (HBS-P buffer, Biacore) and dissociation was monitored for $15 \mathrm{~min}$. Regeneration was achieved with $\mathrm{NaOH}(4.5 \mathrm{mmol} / \mathrm{L})$ after each cycle. The resulting sensorgrams were fitted using BiaEval 4.1 software (Biacore) and for dissociation constant $\left(K_{d}\right)$ calculations, a GY785 molecular weight of $1400000 \mathrm{~g} / \mathrm{mol}$ was used.

\subsection{Synthesis of Si-HPMC/GY785 hydrogel}

The Si-HPMC was synthesized by grafting $0.5 \%$ of silicium in weight on to HPMC $\left(\mathrm{E} 4 \mathrm{M}^{\circledR}\right)$ in heterogeneous medium, as previously described (Xavier Bourges, et al. 2002). Si-HPMC powder $(3 \%, \mathrm{w} / \mathrm{v})$ was solubilized in $0.2 \mathrm{M} \mathrm{NaOH}$ under constant stirring for $48 \mathrm{~h}$. The solution was then sterilized by steam $\left(121^{\circ} \mathrm{C}, 30 \mathrm{~min}\right)$. For the production of Si-HPMC/GY785, sterile GY785 polysaccharide was mixed with the sterile Si-HPMC (3\% w/v) basic solution at the concentration of $10 \mathrm{mg} / \mathrm{mL}(1 \% \mathrm{w} / \mathrm{v})$ in Si-HPMC. The resulting Si-HPMC/GY785 (3\%/1\% w/v) mixture was left under mild rotatory stirring for $12 \mathrm{~h}$ to dissolve the GY785. To allow the formation of a reticulated hydrogel, 1 volume of Si-HPMC (3\% w/v) or Si-HPMC/GY785 (3\%/1\% w/v) solution was mixed with 0.5 volume of $0.26 \mathrm{M}$ HEPES buffer as previously described (Rederstorff, et al. 2011, Vinatier, et al. 2005). The final products consisted of hydrogels $(\mathrm{pH}=7.4)$ containing Si-HPMC $(2 \% \mathrm{w} / \mathrm{v})$ with or without GY785 $(0.67 \% \mathrm{w} / \mathrm{v})$ respectively named Si-HPMC and Si-HPMC/GY785. In the same manner, a hydrogel 
was produced with HPMC in order to obtain a Si-HPMC/HPMC (2/0.67) hydrogel used as a control of macromolecular incorporation of GY785 for the rheological experiments.

\subsection{Animals and surgical procedures}

All animal handling and surgical procedures were conducted according to European Community guidelines for the care and use of laboratory animals (DE 86/609/CEE) and with the agreement of the "pays de la Loire" ethical committee. Experiments were performed according to Good Laboratory Practices (GLP) at the Experimental Therapeutics Unit at the University of Nantes.

\subsection{Isolation of rabbit articular chondrocytes (RAC)}

Rabbit articular cartilage was harvested from euthanized five-week old New Zealand white rabbits and RAC were isolated by enzymatic digestion as described previously (Ghayor, et al. 2000). The suspended RAC were plated (P0) at a density of $5.10^{4}$ cells $/ \mathrm{cm}^{2}$ in a $75 \mathrm{~cm}^{2}$ culture flask with culture medium. The cells were maintained at $37^{\circ} \mathrm{C}$ in a humidified atmosphere of $5 \% \mathrm{CO}_{2}$ and the culture medium was changed every 2-3 days. Cultured RAC were passaged up to four times. RAC from passages 1, 3 and 4 were frozen in Trizol ${ }^{\circledR}$ for subsequent real-time PCR analysis.

\subsection{Cytocompatibility of Si-HPMC/GY785 hydrogel}

Briefly, RAC were allowed to attach in 24-well plates at a final density of $1.10^{4}$ cells $/ \mathrm{cm}^{2}$. After $24 \mathrm{~h}$, the culture medium was removed and $500 \mu \mathrm{l}$ of Si-HPMC/GY785 were added to each well. Samples were incubated at $37^{\circ} \mathrm{C}$ for $1 \mathrm{~h}$ before adding $1 \mathrm{~mL}$ of culture medium. As a control, RAC were also cultured without Si-HPMC/GY785 and Si-HPMC (CT) or in the presence of actinomycin-D $(5 \mu \mathrm{g} / \mathrm{mL})$, an inhibitor of RNA polymerase (Vinatier, et al. 2007), which was used as a potent inducer of cell death. 
After 1, 2, and 3 days, the hydrogels and culture media were removed and Cell viability was measured using an MTS assay as described elsewhere (Vinatier, et al. 2005). Results were expressed as relative MTS activity as compared to control conditions (cells cultured without Si-HPMC/GY785 and Si/HPMC).

Cell proliferation was evaluated by Trypan blue exclusion dye experiments in conditions as described above. After each indicated time, the hydrogel and culture medium were removed and the cells were detached by adding trypsin/EDTA for 2 min. The suspended cells were transferred into fresh culture medium and counted after Trypan blue staining. Results were expressed as the total number of living cells per well.

\subsection{Three-dimensional culture of rabbit articular chondrocytes (RAC)}

RAC (freshly isolated or dedifferentiated (P2)) were gently mixed with SiHPMC/GY785 or Si-HPMC hydrogel (prepared as described previously), at a density of $1.10^{6}$ cells $/ \mathrm{mL}$. Hybrid constructs were then seeded in 12-well plates $(1.5 \mathrm{~mL} /$ well $)$ and incubated at $37^{\circ} \mathrm{C}$ and $5 \% \mathrm{CO}$. After $1 \mathrm{~h}$ incubation, $2 \mathrm{~mL}$ of culture medium was added to each well and changed every 2-3 days. Cell viability was evaluated after 3 weeks. RAC phenotype and the production of sulphated glycosaminoglycans (GAG) and type II collagen were assessed after 3 weeks.

Cell viability in the three-dimensional cultures was visualized using a live and dead cytotoxic assay as previously described (Rederstorff, et al. 2011). The RAC imaging was performed using a confocal laser-scanning microscope (Nikon D-eclipse C1). 
Hybrid constructs associating RAC and hydrogels at 3 weeks were fixed in formol over $1 \mathrm{~h}$ and embedded into $2 \%$ agarose solution before embedding in paraffin. Serial sections of each paraffin block $(5 \mu \mathrm{m}$ thickness $)$ were made and processed for histological staining with alcian blue and immunostained for type II collagen (antirabbit type II collagen 1:100) as described elsewhere (Merceron, et al. 2011). Immunopositive cells showed brown staining with type II collagen antibody.

For the real-time PCR analysis, total RNA was extracted using trizol ${ }^{\circledR}$ reagent in accordance with the manufacturer's instructions. After DNase I digestion, RNA was quantified using a UV-spectrophotometer (Nanodrop NND-1000, Labtech, France). One microgram of RNA per sample was reverse-transcribed using the superscript III kit in a total volume of $20 \mu \mathrm{L}$. Complementary DNA (cDNA) was amplified in a total volume of $25 \mu \mathrm{L}$ PCR reaction mix containing $12.5 \mu \mathrm{L}$ of Brillant ${ }^{\circledR} \mathrm{SYBR}^{\circledR}$ Green Master Mix (1X) and $30 \mathrm{nM}$ of SYBR green reference dye. The sequence and concentration of each primer set are shown in Table 1. The real time polymerase chain reaction was carried out in a MX3000P ${ }^{\circledR}$ real-time PCR system (Stratagene) under the following conditions: $10 \mathrm{~min}$ at $95^{\circ} \mathrm{C}$ followed by 40 cycles of $30 \mathrm{~s}$ at $60^{\circ} \mathrm{C}$ and $30 \mathrm{~s}$ at $72^{\circ} \mathrm{C}$ as previously described (Merceron, et al. 2010). The efficiency and specificity of each primer set was confirmed with standard curves of cycle threshold $(\mathrm{Ct})$ values versus serial dilution of total RNA and melting profile evaluation. Cycle thresholds were normalized to GAPDH to control for cDNA differences. The results are reported as fold change in gene expression relative to control conditions. 


\subsection{In vivo tissue formation}

\subsubsection{Implantation:}

To investigate whether constructs associating RAC with Si-HPMC/GY785 or SiHPMC allow the in vivo formation of cartilaginous tissue, constructs were implanted into subcutaneous pockets of 4-week-old nude mice (Swiss nude mice, Janvier, France). $5.10^{5}$ freshly isolated RAC were gently mixed with $250 \mu \mathrm{L}$ of Si-HPMC/GY785 or SiHPMC hydrogel prior to crosslinking and implanted subcutaneously into nude mice as previously described (Vinatier, et al. 2007). Si-HPMC/GY785 and Si-HPMC without cells were also implanted and used as negative controls. Each condition was tested in quadruplicate and 12 animals were implanted ( 2 implants per animal). After 3 weeks, the mice were sacrificed and the implants were recovered and processed histologically as described earlier. In addition, serial sections of each paraffin block ( $5 \mu \mathrm{m}$ thickness) were immunostained for type I collagen (anti-rabbit type I collagen 1:100)(Merceron, et al. 2011). Immunopositive cells showed brown staining with type I collagen antibody. Rabbit growing bone was used as a positive control for type I collagen immunostaining.

\subsubsection{Type I collagen riboprobe preparation.}

Total RNAs from MC3T3-E1 cells were extracted withTrizolC. One $\mu \mathrm{g}$ of total RNA was reverse transcribed during $60 \mathrm{~min}$ at $42^{\circ} \mathrm{C}$ using 200 units of Superscript II. An equivalent of 100ng of total RNA reverse transcribed was used for PCR using the following primers containing the $\mathrm{T} 3$ or $\mathrm{T} 7$ promoter sequences: Colla1-T3 forward primer, 5'-GAGAATTAACCCTCACTAAAGGGGAGCGGAGAGTACTGGATCG-3' and Col1a1-T7 reverse primer 5'-GAGTAATACGACTCACTATAGGGGGTGGAG GGAGTTTACACGA-3', localized on exon 48 and 50 respectively. The T3- and T7- 
tailed 598-bp-long ${ }^{2} \mathrm{PCR}$ fragment was purified and used as template to synthesize sense and antisense digoxigenin-11-UTP-labeled RNA probes that were prepared using a DIG RNA labelling kit according to the manufacturer's instructions.

\subsubsection{In situ hybridization.}

Tissues were fixed in neutral buffered formalin, embedded in paraffin and sectioned $(4 \mu \mathrm{m})$. Non-radioactive in situ hybridization was adapted from Moorman A.F.M. et al.(Moorman, et al. 2001) Briefly, sections were deparaffinized, treated with $10 \mu \mathrm{g} / \mathrm{mL}$ of proteinase $\mathrm{K}$ for $15 \mathrm{~min}$ at $37^{\circ} \mathrm{C}$, followed by three washes with PBS-tween $0,1 \%$ (PBST). Sections were then re-fixed for $20 \mathrm{~min}$ in $4 \%$ paraformaldehyde dissolved in PBS and washed three times in PBST for $5 \mathrm{~min}$ before being acetylated $(0.1 \mathrm{M}$ triethanolamine with $0.25 \%$ acetic anhydride) for $10 \mathrm{~min}$. Sections were pre-hybridized for $1 \mathrm{hr}$ at $60^{\circ} \mathrm{C}$ and then hybridized overnight at $60^{\circ} \mathrm{C}$. After hybridization sections were rinsed in $2 \mathrm{X} \mathrm{SSC}, \mathrm{pH} 4.5$, washed three times for $30 \mathrm{~min}$ at $60^{\circ} \mathrm{C}$ in $50 \%$ formamide/2X SSC, $\mathrm{pH} 4.5$, followed by three 5-min washes in PBST. Probe bound to the section was immunologically detected using sheep anti-digoxigenin Fab fragment covalently coupled to alkaline phosphatase and NBT/BCIP as chromogenic substrate, essentially according to the manufacturer's protocol (Roche).

The mouse Collal riboprobe was used and demonstrated to hybridize specifically with the rabbit Colla1 transcripts (Fig. 5, C).

\subsection{Rheological characteristics}

One ml of Si-HPMC or Si-HPMC/GY785 or Si-HPMC/HPMC (used as a control of macromolecular incorporation) were allowed to reticulate in 12-well plates. Samples were incubated at $37^{\circ} \mathrm{C}$ for $1 \mathrm{~h}$ before adding $2 \mathrm{ml}$ of DMEM supplemented with $10 \%$ 
FCS, 1\% penicillin/streptomycin and 1\% L-glutamine (culture medium). The plates were maintained at $37^{\circ} \mathrm{C}$ in a humidified atmosphere and with medium change every 2 days. After 3 weeks, oscillatory measurements were performed at $25^{\circ} \mathrm{C}$ on Si-HPMC, Si-HPMC/GY785 or Si-HPMC/HPMC using the Haake MARS rheometer (ThermoHaake ${ }^{\circledR}$, Germany) with titanium plate geometry (20 mm diameter (PP20 Ti), plate with $3 \mathrm{~mm}$ gap) with a homemade device for direct measurement inside the 12 well plates. We applied a $0.2 \mathrm{~N}$ normal force on the hydrogels before the measurements. The complex viscosity $\left(\eta^{*}\right)$ was determined as a function of stress under conditions of linear viscoelastic response in the 0.1 to $5 \mathrm{~Pa}$ range, at a constant oscillation frequency $(\omega)(1 \mathrm{~Hz})$. The storage $\left(G^{\prime}\right)$ and loss $\left(G^{\prime \prime}\right)$ moduli were measured within the linear viscoelastic region.

\subsection{Statistical analysis}

Results are expressed as mean \pm SEM of triplicate determinations. The comparative studies of means were performed using one-way ANOVA followed by a post hoc test with statistical significance set at $p<0.001$. 


\section{Results}

\subsection{Interactions between GY785 and growth factors}

GAG of the extracellular matrix are well known to modulate the activity of a large panel of biological factors. Whether an exogenous GAG, such as GY785, may interact with chondrogenic factors have however not yet been deciphered. To address this issue, we embarked on a set of surface plasmon resonance experiments with two chondrogenic factors namely TGF- $\beta 1$ and BMP-2. To investigate whether these growth factors can specifically interact with GY785, quantitative measurements of their potential physical interaction were performed by surface plasmon resonance analysis. Growth factors were immobilized on the chip and increasing concentrations of GY785 polysaccharide were injected over the chip surface. The results expressed in response units were recorded for each analyte concentration and a $K_{d}$ was calculated. Our biacore data indicate that GY785 polysaccharide was able to strongly bind immobilized TGF- $\beta$ and BMP-2. The binding affinity of GY785 for BMP-2 was about ten-fold higher than for TGF-ß1, with a respective $K_{d}$ of $2.27 .10^{-8}$ and $5.08 .10^{-7} \mathrm{M}$. These results indicate that GY785 can specifically bind BMP-2 and TGF- $\beta 1$.

\subsection{Cytocompatibilty of Si-HPMC/GY785 hydrogels}

To determine whether the addition of GY785 to Si-HPMC hydrogel was cytotoxic or not, the viability and proliferation of primary rabbit articular chondrocytes (RAC) cultured in contact with Si-HPMC/GY785 was examined through measurement of MTS activity and cell counting after 24, 48 and $72 \mathrm{~h}$ of culture. Si-HPMC hydrogel was used as a positive control. The results (Fig. 1 A) show that Si-HPMC and Si-HPMC/GY785 did not affect the MTS activity of RAC at $24 \mathrm{~h}$. After $48 \mathrm{~h}$ of culture, the presence of Si- 
HPMC and Si-HPMC/GY785 slightly reduced the MTS activity of RAC by respectively 20 and $30 \%$. However, after $72 \mathrm{~h}$ there was no significant difference between the MTS activity of RAC cultured in the presence or in the absence of the two different hydrogels. On the contrary, actinomycin-D treatment reduced the MTS activity of RAC by nearly $70 \%$ as after just $24 \mathrm{~h}$ of treatment.

Cell counting experiments (Fig 1 B) produced similar results. After 72h, no significant difference in cell number was observed between cells cultured in contact with SiHPMC/GY785 and cells cultured in control conditions or in contact with Si-HPMC alone.

Taken together, these results indicate that GY785 incorporated to Si-HPMC has neither stimulatory nor adverse effects on RAC viability and proliferation compared to Si-HPMC hydrogel.

To investigate if the presence of GY785 within Si-HPMC influence its 3-dimensional cytocompatibility, the viability of RAC in three-dimensional culture in an SiHPMC/GY785 hydrogel was visualized using confocal microscopy after 3 weeks of culture (Fig 1 C). Cells were stained with calcein AM and "EthD-1" which respectively label living cells green and dead cells red. As shown in Fig 1C, RAC 3-dimensionally (3D) cultured in Si-HPMC/GY785 were green and organized in nodular structures as observed with RAC cultured in Si-HPMC alone. The absence of red fluorescence indicates the absence of dead cells in the Si-HPMC/GY785. These results show that RAC retained their viability and organized into nodular structures when threedimensionally cultured in Si-HPMC/GY785 hydrogel indicating that GY785 incorporation didn't modify the 3D cytocompatibility of Si-HPMC. 


\subsection{Maintenance of chondrocyte phenotype in three-dimensional cultures}

To assess whether GY785 enrichment could act on the ability of Si-HPMC hydrogel to maintain chondrocytic phenotype in a three-dimensional culture, RAC were cultured for 3 weeks either in two dimensions (2D) without hydrogels or in three dimensions (3D) in Si-HPMC/GY785 hydrogel or Si-HPMC alone as a control. The expression of transcripts coding for type I collagen (dedifferentiation marker), type II collagen and aggrecan were evaluated with real time PCR (Fig. 2 A). 2D-cultured RAC showed an expression of type II collagen and aggrecan of 10- and 14.3-fold lower than primary RAC (P0) respectively. RAC cultured in 3D in Si-HPMC/GY785 expressed levels of type II collagen and aggrecan transcripts that were significantly higher of approximately 5- and 19-fold respectively when compared to 2D RAC and 2,7- and 3-fold respectively as compared to Si-HPMC 3D-cultured RAC. Likewise, RAC cultured in 3D in SiHPMC alone exhibited an expression of type II collagen and aggrecan 2- and 7-fold higher than primary RAC. Whereas the expression of type I collagen in 2D RAC (fig 2 A) was more than 3 times higher than that of RAC (P0), 3D cultured RAC in SiHPMC/GY785 exhibited a barely detectable increase in type I collagen expression. RAC cultured in 3D in Si-HPMC did not show any increase of the expression of type I collagen as compared to primary RAC (P0).

These results exhibit that GY785 enrichment of Si-HPMC hydrogel seems to increase its ability in maintaining high level of type II collagen and Aggrecan mRNA synthesis. These results taken as a whole also demonstrate the capability of SiHPMC/GY785 hydrogels to maintain a differentiated chondrocyte-like phenotype in RAC. 
To further investigate the ability of Si-HPMC/GY785 to maintain a differentiated chondrocytic phenotype, the effect of 3D culture in Si-HPMC/GY785 on sulphated GAG and immunostaining for type II collagen were performed on RAC cultured in 3D in Si-HPMC/GY785 or Si-HPMC for 3 weeks. Under these conditions (Figure 2 B), and despite an Alcian Blue positive staining of cell-free GY785 containing Si-HPMC (data not shown), both hydrogels exhibited positive Alcian blue staining and type II collagen immunostaining. These results show an accumulation of sulphated GAG and type II collagen in the extracellular matrix surrounding the RAC nodules formed in $3 \mathrm{D}$ culture in Si-HPMC/GY785 hydrogels as observed with Si-HPMC alone. However, SiHPMC/GY785 hydrogel allows the formation of broadest alcian blue and type II collagen positive RAC nodules compared to Si-HPMC hydrogel.

Taken together, these results indicate that Si-HPMC/GY785 hydrogel supported the 3D culture of functionally competent RAC able to produce type II collagen and sulphated GAG.

\subsection{Dedifferentiation of RAC in $2 \mathrm{D}$ cultures and redifferentiation in $3 \mathrm{D}$ cultures}

It is well known that dedifferentiated articular chondrocytes are able to retrieve a chondrocytic phenotype when replaced in a suitable three-dimensional environment (Chen, et al. 2003, Miot, et al. 2005, Vinatier, et al. 2007). We therefore sought to evaluate whether this phenomenon also occurs with RAC initially cultured in $2 \mathrm{D}$ and thereafter placed in 3D in Si-HPMC/GY785 hydrogels. For this experiment, freshly isolated RAC were first dedifferentiated by culturing them into monolayer for four passages. Expressions of transcripts coding for type I and II collagen and aggrecan were evaluated using real time PCR (Fig. 3 A). When the RAC were passaged, they showed 
an approximately 4-fold decrease in type II collagen expression at passage 2 (P2) and 16-fold decreased at P4 as compared to the primary RAC (P0). Likewise, aggrecan expression exhibited a 3-fold and 4.5-fold decrease after passages 2 and 4 respectively (Fig. 3 A, P2, P4). On the contrary, the expression of type I collagen transcript increased dramatically about 7 times and 10 times after respectively 2 and 4 passages in monolayer culture (Fig. 3 A). These results show that the RAC underwent a dedifferentiation process as early as passage 2 (P2).

Therefore, to investigate whether Si-HPMC/GY785 allows the recovery of a chondrocytic phenotype, dedifferentiated RAC P2 were placed for 3 additional weeks either in 3D culture in Si-HPMC/GY785 or Si-HPMC or in a 2D culture. Real-time PCR analysis revealed that after 3 weeks, the RAC cultured in 3D in Si-HPMC/GY785 exhibited a 28-fold increase in type II collagen expression and a 75 -fold increase in aggrecan expression as compared to the RAC P2 (Figure $3 \mathrm{~B}$ ). On the contrary, the RAC cultured in 2D for the same additional duration failed to exhibit any significant increase in type II collagen and aggrecan transcripts. Interestingly, expression of the dedifferentiation marker type I collagen after 3 weeks in $3 \mathrm{D}$ culture in $\mathrm{Si}$ HPMC/GY785 was 3.4 times less than that measured in the RAC P2 (Fig. 3 B). 2D RAC exhibited a 2-fold increase in type I collagen expression as compared to RAC P2. When compared to the control hydrogel (Si-HPMC), the RAC cultured in SiHPMC/GY785 exhibited a 2-fold increase in expression of the transcript coding for type II collagen and aggrecan. These results suggest that the recovery of the chondrocytic phenotype is improved in Si-HPMC/GY785 hydrogel compared to SiHPMC hydrogel alone. 


\subsection{In vivo tissue formation}

To investigate whether the addition of GY785 within Si-HPMC hydrogel makes possible the formation of cartilaginous tissue in vivo, constructs associating freshly isolated RAC with Si-HPMC/GY785 or Si-HPMC hydrogels were implanted subcutaneously into nude mice. After 3 weeks, histological examinations (Fig. 4 A) showed the formation of chondroid nodules with RAC associated with SiHPMC/GY785 as well as with the control hydrogel (Si-HPMC). Nodules formed by RAC were positively stained with Alcian blue (Fig. 4 A), suggesting the production of an extracellular matrix-containing GAG. In addition, these nodules were positively stained for type II collagen (Fig. 4 A). On the contrary, type I collagen immunostaining remained barely detectable in both conditions (Fig. 4 B left and middle panel). As a control, rabbit growing bone exhibited an intense type I collagen immunostaining (Fig. 4 B right panel)

As an additional control, Si-HPMC and Si-HPMC/GY785 alone showed neither the formation of nodular structure nor the presence of an extracellular matrix despite a positive Alcian blue staining in cell-free Si-HPMC/GY785 hydrogel (data not shown).

To confirm the absence of type I collagen immunostaining, in situ hybridization for type I collagen transcripts were then performed. In situ hybridization indicates that mRNA coding for type I collagen remains at a barely detectable level in GY785 reinforced SiHPMC and Si-HPMC hydrogels (Fig. $4 \mathrm{C}$ left and middle panel). As a control, ISH for type I collagen in rabbit growing bone was found to be intensely positive (Fig. $4 \mathrm{C}$ right panel). 
These results indicate that Si-HPMC enriched with GY785 makes possible the formation of cartilaginous tissue in vivo with freshly isolated RAC.

\subsection{Rheological characteristics}

To evaluate if GY785 incorporation modify the viscoelastic properties of Si-HPMC, an oscillatory measurement was performed to evaluate the elastic modulus (G') and viscous modulus (G') (Fig 5). The Si-HPMC/GY785 showed a G' of about $723+/-171$ $\mathrm{Pa}$ and a G" of about $96+/-37 \mathrm{~Pa}$. Si-HPMC and Si-HPMC/HPMC exhibited the same G' with values of about $398+/-65 \mathrm{~Pa}$ and $324+/-52 \mathrm{~Pa}$ and G" of about 28+/-6 Pa and 22+/-5 Pa respectively.

These results indicate that Si-HPMC/GY785 hydrogel has higher stiffness than SiHPMC and Si-HPMC/HPMC whereas Si-HPMC and Si-HPMC/HPMC have similar stiffness. 


\section{Discussion}

Today, regenerative medicine is moving towards the development of less and less invasive surgical techniques with the aim of reducing morbidity and hospitalization time. From this point of view, injectable hydrogels appear to be promising (Cushing and Anseth 2007). The main challenge in developing new hydrogels for cartilage tissue engineering is to allow the viability and differentiation of cells within the hydrogel.

Marine-based polysaccharides (Colliec-Jouault, et al. 2004) that exhibit structural analogies with glycosaminoglycans (GAGs) are well acknowledged as molecules influencing cell proliferation, differentiation and adhesion (Chiu, et al. 2010). GAGs are long known to be able to bind and regulate the biological activity of a large number of growth factors through the modulation of their storage, stabilization and degradation in the matrix (Jackson, et al. 1991, Vlodavsky, et al. 2006). To determine whether TGF- $\beta 1$ or BMP-2, two major chondrogenic growth factors, can physically interact with the marine polysaccharides GY785, surface plasmon resonance experiments were performed. Interestingly, our Biacore data demonstrate the existence of a high affinity between GY785 and the chondrogenic factors TGF- $\beta 1$ and BMP-2. These data therefore establish a strong rationale for testing our hypothesis that GY785 could be used to enrich scaffolding biomaterials and improves their potential for engineering cartilage. In this attempt and to propose a three-dimensional scaffolding hydrogel capable of supporting regeneration of functional cartilage, a self-setting cellulosic hydrogel (SiHPMC), widely used for cartilage tissue engineering was chosen (Portron, et al. 2013, Vinatier, et al. 2009). A prerequisite to the use of hydrogels for in vivo experiments is to evaluate their non-cytotoxicity. We were therefore first interested in examining the in vitro cytotoxicity of Si-HPMC/GY785 with rabbit primary articular chondrocytes 
(RAC). We thus focused on a cytotoxicity test with direct contact with RAC (ISO 10993-5: Biological evaluation of medical devices-Part 5: test for in vitro cytotoxicity). Our data indicate that the $\mathrm{Si}-\mathrm{HPMC} / \mathrm{GY785}$ hydrogel is cytocompatible with respect to RAC as evidenced by estimation of mitochondrial dehydrogenase activity (MTS assay) and cell counting (Fig. 1). In addition, the dual fluorescent staining using calcein AM and EthD-1 reagents, revealed that RAC three-dimensionally cultured in SiHPMC/GY785 hydrogel retained their viability and formed nodules (Fig. 1). These results indicate that the $\mathrm{Si}-\mathrm{HPMC} / \mathrm{GY} 785$ hydrogel makes possible the threedimensional culture of RAC without altering their viability and proliferation.

Another characteristic of Si-HPMC hydrogel, shared with many other biomaterials used for cartilage tissue engineering, is its ability to maintain the chondrocytic phenotype in 3 dimensional culture (Vinatier, et al. 2005). Indeed, it is well known that maintaining or recovering a chondrocytic phenotype in a scaffolding hydrogel is a key point for cartilage tissue engineering strategies. Dedifferentiation of chondrocytes, evidenced by a concomitant decrease in type II collagen and aggrecan expression and an increase in type I collagen expression, effectively occurs during in vitro $2 \mathrm{D}$ monolayer expansion (Brodkin, et al. 2004, Schnabel, et al. 2002). In our hands and according to this dedifferentiation process, type II collagen and aggrecan expressions were downregulated along with passages in RAC, whereas that of type I collagen expression increased. However, culturing chondrocytes in three-dimensional scaffolds has been shown to not only prevent, but even to reverse, this phenomenon, allowing dedifferentiated chondrocytes to recover their phenotype (Vinatier, et al. 2005). Accordingly, the expressions of type II collagen and aggrecan are maintained in 3D cultures in Si-HPMC with and without GY785, highlighting their ability to maintain a 
chondrocyte-like phenotype in vitro. Moreover, the GY785 enrichment of Si-HPMC increases of approximately 2- to 3-fold the expression level of type II collagen and aggrecan when compared to Si-HPMC alone. These results were further confirmed by the production of GAG and collagen type II by three-dimensionally cultured RAC in the Si-HPMC/GY785 hydrogel that appears higher than in Si-HPMC.

In addition and of particular interest for cell-based cartilage tissue engineering, the SiHPMC hydrogel also induces the recovery of a chondrocytic phenotype of previously dedifferentiated RAC (P2) as evidenced by the increased expression of type II collagen and aggrecan while expression of type I collagen is decreased. Further, GY785 enrichment of Si-HPMC hydrogel improves this recovery of chondrocytic phenotype as evidenced by the 2-fold increase in type II collagen and Aggrecan expression as compared to Si-HPMC alone. It therefore seems reasonable to speculate that SiHPMC/GY785 not only make possible the three-dimensional culture of phenotypically stable chondrocytes but also allows the recovery of such a chondrocytic phenotype in an upper manner than Si-HPMC alone. This result strongly suggests that GY785 positively altered the biological activity of Si-HPMC hydrogel.

To address the in vivo potential of Si-HPCM/GY785 as a new hydrogel for cartilage engineering, we secondly embarked on a preliminary animal experiment in a welldocumented model of tissue engineering, the subcutis of nude mice. Hybrid systems associating chondrocytes (RAC) with Si-HPMC/GY785 were implanted into subcutaneous sites in the back of nude mice. Si-HPMC associated with RAC was used as a control. After 3 weeks of implantation, constructs associating RAC with SiHPMC/GY785 showed the formation of nodules with an extracellular matrix containing 
sulphated GAG and type II collagen. Interestingly, type I collagen expression at both the mRNA and protein levels remained barely detectable, suggesting that cells do not likely undergo any dedifferentiation process.

Given that GY785 polysaccharide is a high molecular weight macromolecule, incorporating GY785 into Si-HPMC hydrogel certainly modified its mechanical properties. To address this issue, the rheological properties of $\mathrm{Si}-\mathrm{HPMC} / \mathrm{GY785}$, SiHPMC and Si-HPMC/HPMC hydrogels were determined and indicates that G' was higher than G" (Fig. 5) for the three hydrogels, thereby confirming that these hydrogels are solid after 3 weeks. The G' of Si-HPMC/GY785 is approximately 2 times higher than the one of Si-HPMC or Si-HPMC/HPMC, showing that enrichment of Si-HPMC with GY785 increased its stiffness (Rederstorff, et al. 2011). The storage modulus G' was also linked to the network density in polysaccharide hydrogels (Moe, et al. 1992). In our study, the G' value revealed a higher network density with the adjunction of GY785 polysaccharides (Fig. 5). The increased stiffness and network density in GY785enriched Si-HPMC may explain the enhancement of chondrocytes phenotype in this hydrogel compared to Si-HPMC alone. Indeed, these mechanical parameters have previously been shown to influence chondrocyte phenotype (Brodkin, et al. 2004, SanzRamos, et al. 2013) as well as direct mesenchymal stem cell differentiation (Discher, et al. 2005, Engler, et al. 2006).

\section{Conclusions}

In conclusion, our results show that GY785 physically interacts with some chondrogenic factors. Si-HPMC doped with GY785 is cytocompatible and competent for the three-dimensional culture of chondrocytes capable of producing cartilage ECM 
proteins in vitro. GY785 enriched Si-HPMC interestingly supports the recovery of a chondrocytic phenotype in dedifferentiated articular chondrocytes. In vivo, this doped hydrogel allows the synthesis of a cartilage-like ECM by chondrocytes in subcutis of nude mice. This site is however rather far from an articular cartilage situation due to the lack of resident endogenous growth factors. Regarding the growth factors-interacting properties of GY785, the preclinical relevance of GY785 doped hydrogel will be given further attention in adapted animal models of articular cartilage defects where growth factors, notably TGF- $\beta$, are found in large amount (Goldring, et al. 2006).

\section{Acknowledgements:}

E.R. received a fellowship from the "Region Pays de la Loire", through the Bioregos grant. This study was supported by grants from the ANR TecSan "chondrograft", the Arthritis Fondation Courtin and the "Société Française de Rhumatologie". The authors would also like to thank S. Laib, M. Masson, C. Colombeix, C. Sinquin and J. Ratiskol. 


\section{References:}

Bonzani IC, George JH and Stevens MM 2006, Novel materials for bone and cartilage regeneration, Curr Opin Chem Biol, 10: 568-75.

Bourges X, Weiss P, Coudreuse A, et al. 2002, General properties of silated hydroxyethylcellulose for potential biomedical applications, Biopolymers, 63: 232-8.

Bourges X, Weiss P, Daculsi G, et al. 2002, Synthesis and general properties of silatedhydroxypropyl methylcellulose in prospect of biomedical use, Advances in Colloid and Interface Science, 99: 215-228.

Brodkin KR, Garcia AJ and Levenston ME 2004, Chondrocyte phenotypes on different extracellular matrix monolayers, Biomaterials, 25: 5929-38.

Casu B and Lindahl U 2001, Structure and biological interactions of heparin and heparan sulfate, Adv Carbohydr Chem Biochem, 57: 159-206.

Chen G, Sato T, Ushida T, et al. 2003, Redifferentiation of dedifferentiated bovine chondrocytes when cultured in vitro in a PLGA-collagen hybrid mesh, FEBS Lett, 542: 95-9.

Chiu LH, Chen SC, Wu KC, et al. 2010, Differential effect of ECM molecules on re-expression of cartilaginous markers in near quiescent human chondrocytes, J Cell Physiol:

Colliec-Jouault S, Zanchetta P, Helley D, et al. 2004, Les polysaccharides microbiens d'origine marine et leur potentiel en thérapeutique humaine, Pathologie Biologie, 52: 127-130.

Cushing MC and Anseth KS 2007, Materials science. Hydrogel cell cultures, Science, 316: 11334.

Dehne T, Schenk R, Perka C, et al. 2010, Gene expression profiling of primary human articular chondrocytes in high-density micromasses reveals patterns of recovery, maintenance, reand dedifferentiation, Gene, 462: 8-17.

Demoor M, Ollitrault D, Gomez-Leduc T, et al. 2014, Cartilage tissue engineering: Molecular control of chondrocyte differentiation for proper cartilage matrix reconstruction, Biochim Biophys Acta, 1840: 2414-2440.

Discher DE, Janmey P and Wang YL 2005, Tissue cells feel and respond to the stiffness of their substrate, Science, 310: 1139-43.

Engler AJ, Sen S, Sweeney HL, et al. 2006, Matrix elasticity directs stem cell lineage specification, Cell, 126: 677-89.

Ghayor C, Herrouin JF, Chadjichristos C, et al. 2000, Regulation of human COL2A1 gene expression in chondrocytes. Identification of C-Krox-responsive elements and modulation by phenotype alteration, J Biol Chem, 275: 27421-38.

Goldring MB, Tsuchimochi K and ljiri K 2006, The control of chondrogenesis, J Cell Biochem, 97: 33-44.

Gomoll AH and Minas T 2014, The quality of healing: articular cartilage, Wound Repair Regen, 22 Suppl 1: 30-8.

Griffith LG and Naughton G 2002, Tissue engineering--current challenges and expanding opportunities, Science, 295: 1009-14.

Guezennec J 2002, Deep-sea hydrothermal vents: a new source of innovative bacterial exopolysaccharides of biotechnological interest?, J Ind Microbiol Biotechnol, 29: 204-8.

Jackson RL, Busch SJ and Cardin AD 1991, Glycosaminoglycans: molecular properties, protein interactions, and role in physiological processes, Physiol Rev, 71: 481-539.

Matou S, Colliec-Jouault S, Galy-Fauroux I, et al. 2005, Effect of an oversulfated exopolysaccharide on angiogenesis induced by fibroblast growth factor-2 or vascular endothelial growth factor in vitro, Biochem Pharmacol, 69: 751-9. 
Merceron C, Portron S, Masson M, et al. 2011, The effect of two and three dimensional cell culture on the chondrogenic potential of human adipose-derived mesenchymal stem cells after subcutaneous transplantation with an injectable hydrogel, Cell Transplant:

Merceron C, Portron S, Vignes-Colombeix C, et al. 2012, Pharmacological modulation of human mesenchymal stem cell chondrogenesis by a chemically oversulfated polysaccharide of marine origin: potential application to cartilage regenerative medicine, Stem cells, 30: 47180.

Merceron C, Vinatier C, Portron S, et al. 2010, Differential effects of hypoxia on osteochondrogenic potential of human adipose-derived stem cells, Am J Physiol Cell Physiol, 298: C355-64.

Miot S, Woodfield T, Daniels AU, et al. 2005, Effects of scaffold composition and architecture on human nasal chondrocyte redifferentiation and cartilaginous matrix deposition, Biomaterials, 26: 2479-89.

Moe ST, Draget KI, Skjåk-Bræk G, et al. 1992, Temperature dependence of the elastic modulus of alginate gels, Carbohydrate Polymers, 19: 279-284.

Moorman AF, Houweling AC, de Boer PA, et al. 2001, Sensitive nonradioactive detection of mRNA in tissue sections: novel application of the whole-mount in situ hybridization protocol, the Journal of Histochemistry and Cytochemistry, 49: 1-8.

Mukaida T, Urabe K, Naruse K, et al. 2005, Influence of three-dimensional culture in a type II collagen sponge on primary cultured and dedifferentiated chondrocytes, J Orthop Sci, 10: 521-8.

Portron S, Merceron C, Gauthier O, et al. 2013, Effects of in vitro low oxygen tension preconditioning of adipose stromal cells on their in vivo chondrogenic potential: application in cartilage tissue repair, PloS one, 8: e62368.

Rederstorff E, Weiss P, Sourice S, et al. 2011, An in vitro study of two GAG-like marine polysaccharides incorporated into injectable hydrogels for bone and cartilage tissue engineering, Acta Biomater, 7: 2119-30.

Sanz-Ramos P, Mora G, Vicente-Pascual M, et al. 2013, Response of sheep chondrocytes to changes in substrate stiffness from 2 to 20 Pa: effect of cell passaging, Connect Tissue Res, 54: 159-66.

Schnabel M, Marlovits S, Eckhoff G, et al. 2002, Dedifferentiation-associated changes in morphology and gene expression in primary human articular chondrocytes in cell culture, Osteoarthritis Cartilage, 10: 62-70.

Steinert AF, Ghivizzani SC, Rethwilm A, et al. 2007, Major biological obstacles for persistent cell-based regeneration of articular cartilage, Arthritis Res Ther, 9: 213.

van Osch GJ, Brittberg M, Dennis JE, et al. 2009, Cartilage repair: past and future--lessons for regenerative medicine, J Cell Mol Med, 13: 792-810.

Vinatier C, Bouffi C, Merceron C, et al. 2009, Cartilage tissue engineering: towards a biomaterial-assisted mesenchymal stem cell therapy, Curr Stem Cell Res Ther, 4: 318-29.

Vinatier C, Gauthier O, Fatimi A, et al. 2009, An injectable cellulose-based hydrogel for the transfer of autologous nasal chondrocytes in articular cartilage defects, Biotechnol Bioeng, 102: 1259-67.

Vinatier C, Magne D, Moreau A, et al. 2007, Engineering cartilage with human nasal chondrocytes and a silanized hydroxypropyl methylcellulose hydrogel, J Biomed Mater Res A, 80: 66-74.

Vinatier C, Magne D, Weiss P, et al. 2005, A silanized hydroxypropyl methylcellulose hydrogel for the three-dimensional culture of chondrocytes, Biomaterials, 26: 6643-51.

Vlodavsky I, Abboud-Jarrous G, Elkin M, et al. 2006, The impact of heparanese and heparin on cancer metastasis and angiogenesis, Pathophysiol Haemost Thromb, 35: 116-27. 
Yamada S and Sugahara K 2008, Potential therapeutic application of chondroitin sulfate/dermatan sulfate, Current drug discovery technologies, 5: 289-301.

Zcharia E, Zilka R, Yaar A, et al. 2005, Heparanase accelerates wound angiogenesis and wound healing in mouse and rat models, FASEB J, 19: 211-21. 


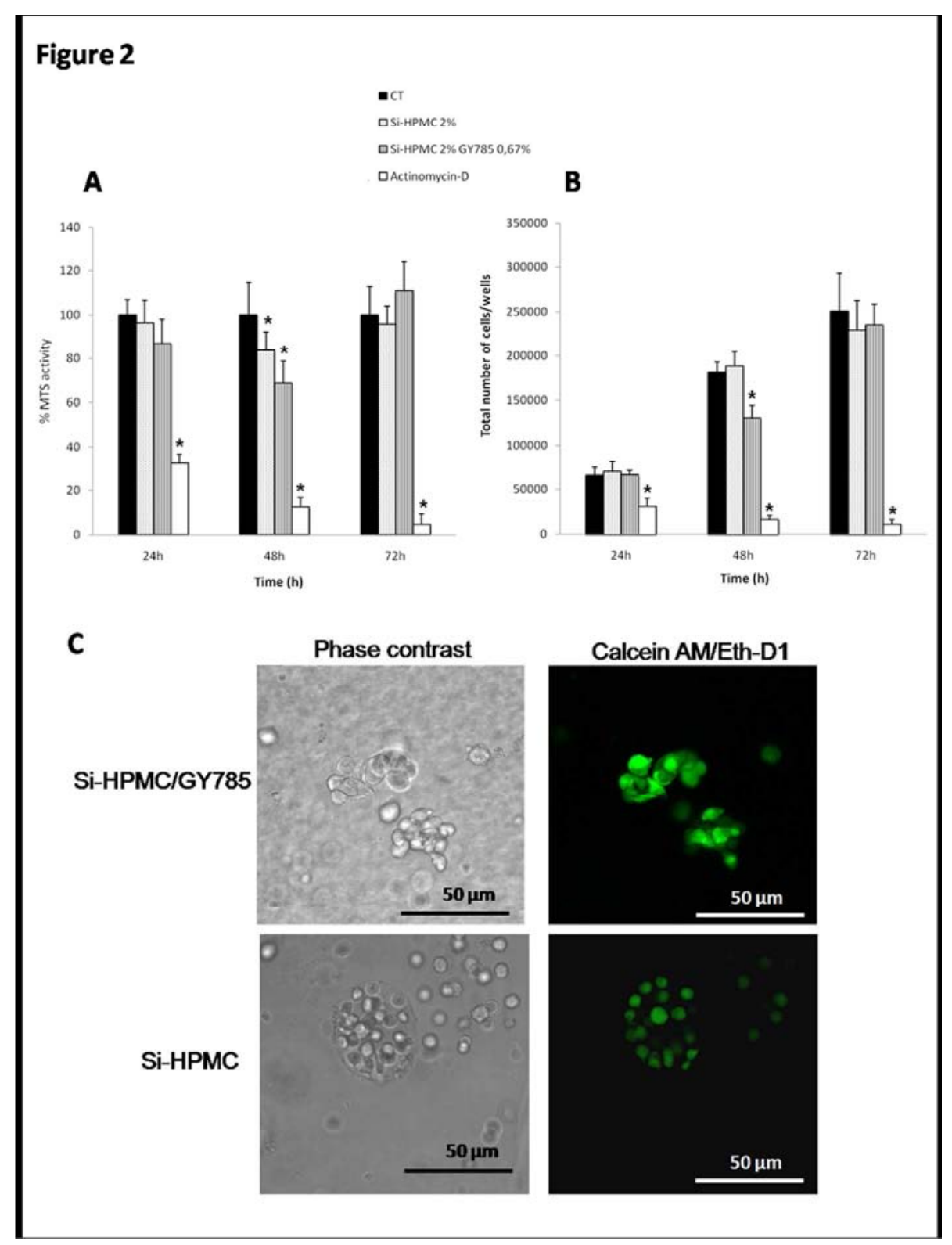

Figure 1: Cytocompatibility of Si-HPMC/GY785 with RAC in two- (2D) and three-dimensional culture (3D). Rabbit articular chondrocytes (RAC) were cultured in 2D on culture plates over $72 \mathrm{~h}$ without hydrogel (positive control, CT) or with actinomycin-D $(5 \mu \mathrm{g} / \mathrm{ml})$ (negative control) or with Si-HPMC $(2 / 0)$ or SiHPMC/GY785 (2/0.67). A) Viability was assessed by MTS activity as indicated in the materials and methods. Results are expressed as relative MTS activity compared with the respective controls. $* p<0.001$ as compared to control conditions. B) Proliferation was assessed by scoring the cells after trypan blue staining. Results are expressed as the total number of cells per well. *p $<0.001$ compared with the respective controls. C) RAC were cultured in 3D for 3 weeks in Si-HPMC/GY785 (2/0.67) or Si-HPMC (2/0) prior to staining with calcein AM and EthD-1. Viability was visualized using confocal microscopy as indicated in the materials and methods. Scale length: $50 \mu \mathrm{m}$. $192 \times 254 \mathrm{~mm}$ ( $300 \times 300$ DPI) 
-2D

ロ3D Si-HPMC/GY785 $(2 / 0,67)$

口3D Si-HPMC (2/0)
B
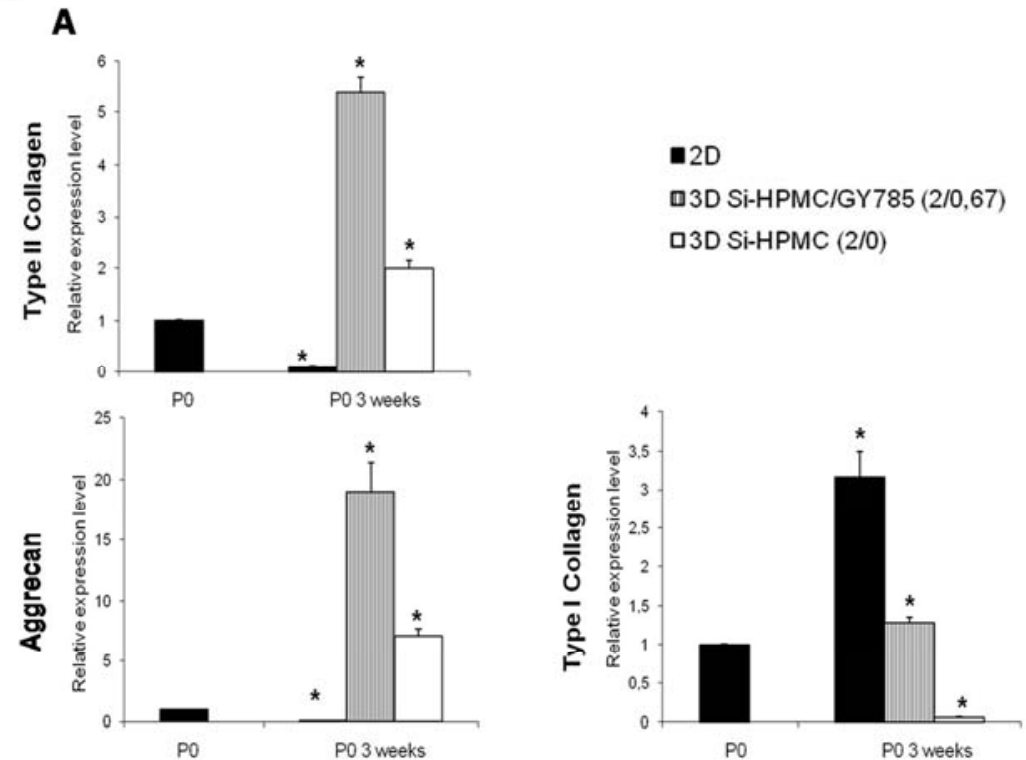

GAG

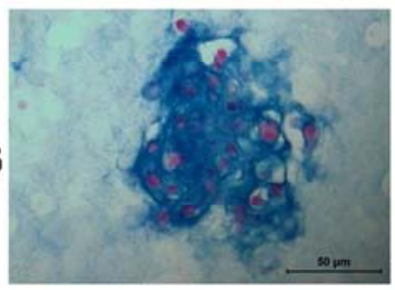

Type II collagen

Si-HPMC/GY785
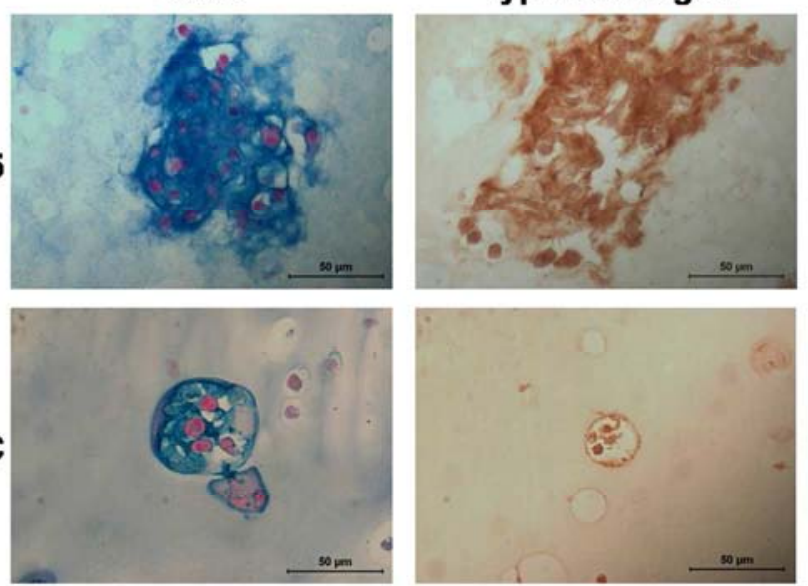

Si-HPMC

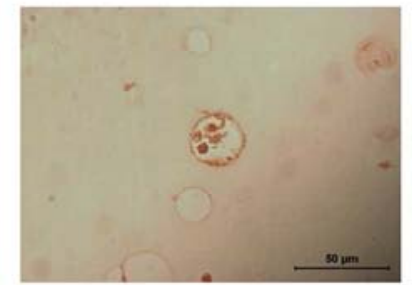

Figure 2: The effects of a 3D culture on the RAC phenotype. A) Maintenance of chondrocyte phenotype in three-dimensional (3D) culture. Total RNA was purified from freshly isolated RAC (P0), from RAC cultured in 2D and RAC cultured in 3D in Si-HPMC/GY785 or Si-HPMC for 3 weeks (P0 3 weeks). Expression of the chondrocytic markers (type II collagen and aggrecan) as well as the dedifferentiation marker (Type I collagen) were investigated using real-time PCR as indicated in the materials and methods. Results are expressed as relative expression levels compared to the control conditions (P0). B) Cartilaginous matrix production by RAC in three-dimensional culture. Rabbit articular chondrocytes were cultured for 3 weeks in 3D in Si-HPMC/GY785 (2/0.67) and Si-HPMC (2/0). Alcian blue staining for Glycosaminoglycans (GAG) and immunohistochemical type II collagen stainings were carried out as detailed in the materials and methods. Samples were observed with a light microscope. Scale length: $50 \mu \mathrm{m}$. $192 \times 255 \mathrm{~mm}(300 \times 300 \mathrm{DPI})$ 


\section{Figure 4}

\section{A}
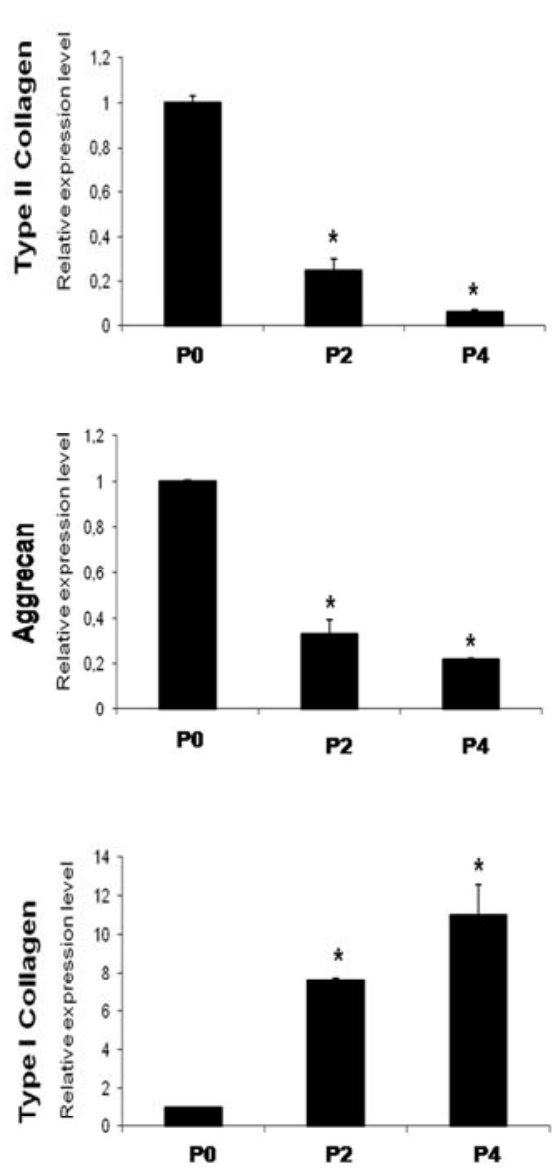

B $\square 3 D$ Si-HPMC/GY785 $(2 / 0,67)$

口3D Si-HPMC (2/0)
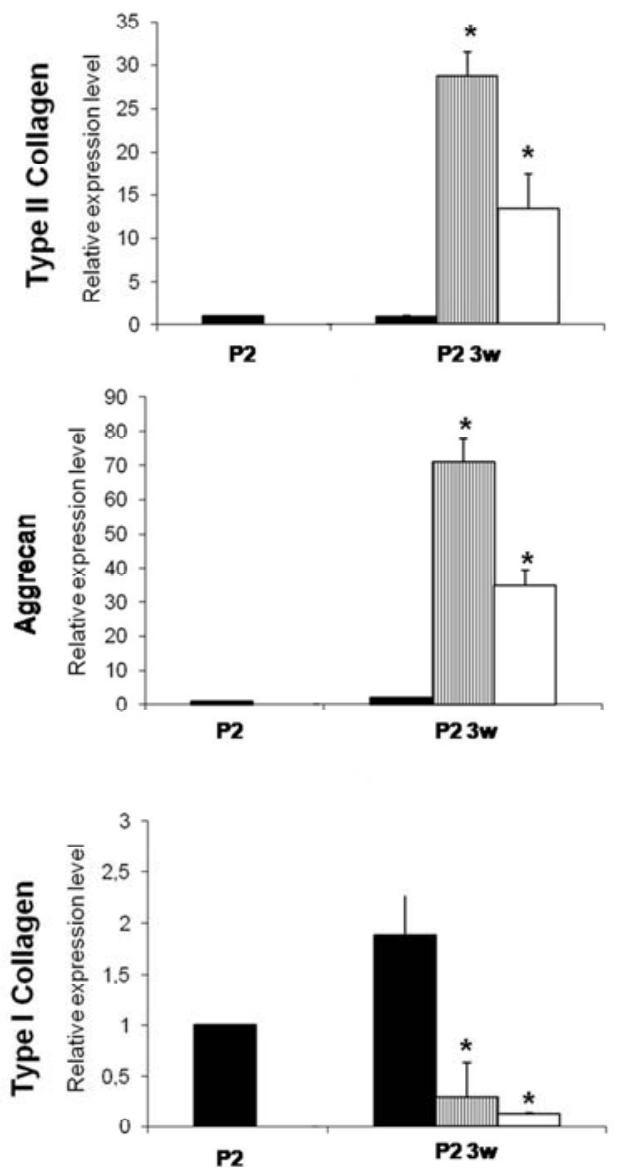

Figure 3: Recovery of a chondrocytic phenotype in dedifferentiated RAC by 3D culture. A) Dedifferentiation of RAC in monolayer culture. Total RNA was purified from freshly isolated RAC (P0), from RAC cultured in two dimensions for 2 (P2) and 4 (P4) passages. Real time PCR analysis for type II collagen and aggrecan as well as type I collagen transcripts were performed using the primers and conditions detailed in the

experimental procedures. Results are expressed as relative expression levels compared to the control conditions P0. * $\mathrm{p}<0.001$ compared to RAC (P0). B) Redifferentiation of RAC in three-dimensional culture. Total RNA was purified from dedifferentiated RAC at passage 2 (P2) and from dedifferentiated RAC cultured for 3 additional weeks (P2 3w) in 3D in Si-HPMC/GY785 (2/0.67) and Si-HPMC (2/0). Real time PCR analysis for type II and I collagen transcripts, as well as aggrecan, were carried out using the primers and conditions detailed in the experimental procedures. Results are expressed as relative expression levels compared to the control conditions (P2). * $\mathrm{p}<0.001$ compared to $\mathrm{P} 2$. $208 \times 240 \mathrm{~mm}(300 \times 300 \mathrm{DPI})$ 
B

\section{Figure 5}

A
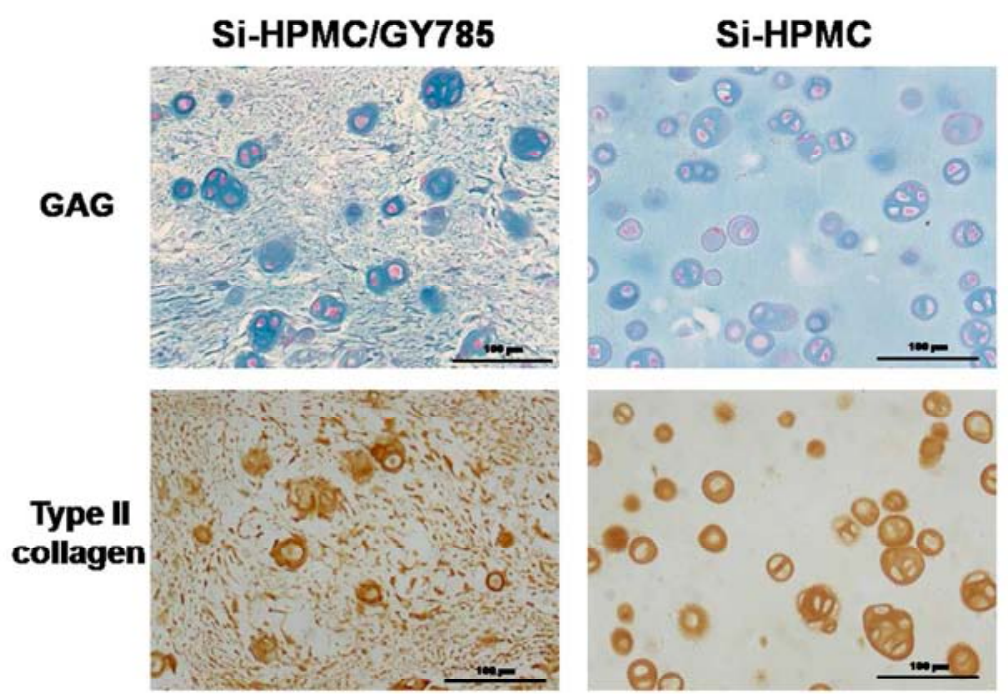

B
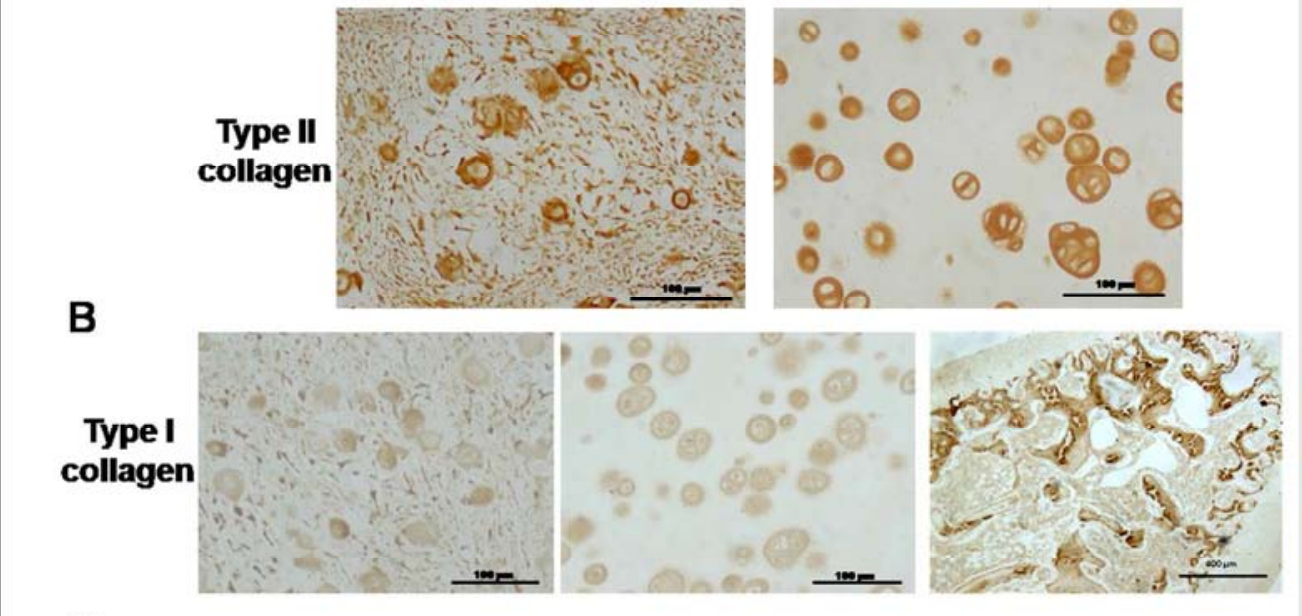

\section{C

Type I
collagen

Figure 4: Histochemical analysis of tissue engineered cartilage. RAC associated with Si-HPMC/GY785 (2/0.67) or Si-HPMC (2/0) were implanted subcutaneously into nude mice and analyzed 3 weeks later. A) Histological sections were stained, as described in the materials and methods, for the presence of GAG (Alcian blue) and type II collagen (Immunostaining). Samples were observed with a light microscope. Scale length: $100 \mu \mathrm{m}$. B) Histological sections were stained, as described in the materials and methods, for the presence of type I collagen (Immunostaining). Samples were observed with a light microscope. Scale length: $100 \mu \mathrm{m}$. Positive control (right panel) was performed with rabbit growing bone. Scale length: $400 \mu \mathrm{m}$.

C) histological sections were processed for in situ hybridization and hybridized with col1A1 riboprobe as described in the materials and methods. Scale length: $100 \mu \mathrm{m}$. Positive control (right panel), was performed with rabbit growing bone. Scale length: $400 \mu \mathrm{m}$ $194 \times 214 \mathrm{~mm}(300 \times 300 \mathrm{DPI})$ 
1

2

3

4

5

6

7

8

9

10

11

12

13

14

15

16

17

18

19

20

21

22

23

24

25

26

27

28

29

30

31

32

33

34

35

36

37

38

39

40

41

42

43

44

45

46

47

48

49

50

51

52

53

54

55

56

57

58

59

60

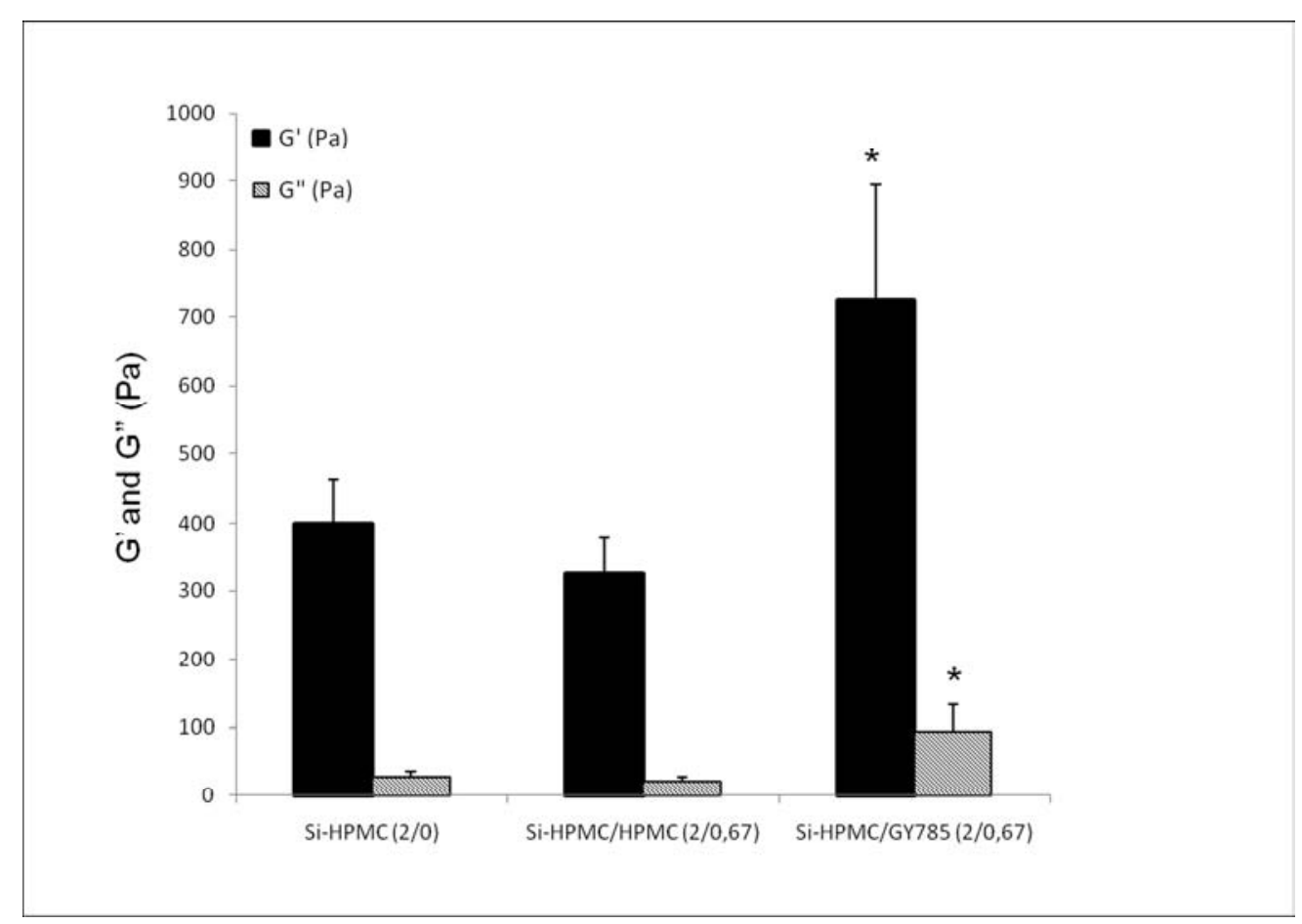

Figure 5: Oscillatory measurements. Oscillatory measurements of the different hydrogels (Si-HPMC, SiHPMC/HPMC (2/0.67) and Si-HPMC/GY785 (2/0.67)) after 3 weeks of storage at $37^{\circ} \mathrm{C}$ in a humid environment. Measurements $(n=3)$ were made directly in 12 multi-well culture plates using a

ThermoHaake ${ }^{\circ}$, (Germany, Mars ${ }^{\mathrm{TM}}$, Titanium plate PP20Ti) with the following parameters: gap $\approx 3 \mathrm{~mm}$, normal force $0.2 \mathrm{~N}, 1 \mathrm{Htz}$, from 0.1 to $5 \mathrm{~Pa}$, at $25^{\circ} \mathrm{C}$. The conservative modulus $\mathrm{G}^{\prime}$ and loss modulus $\mathrm{G}^{\prime \prime}$ are shown in $\mathrm{Pa}$. ${ }^{*} \mathrm{p}<0.001$ compared with the respective controls Si-HPMC and Si-HPMC/HPMC.

$190 \times 134 \mathrm{~mm}(300 \times 300 \mathrm{DPI})$ 
Table 1. Sequences of primer pairs, gene bank accession numbers used for real time RT-PCR analysis and size of PCR products

\begin{tabular}{|c|c|c|c|c|}
\hline Gene & GeneBank No. & Forward primer & Reverse primer & Amplicon size (bp) \\
\hline $\begin{array}{l}\text { Glyceraldehyde 3-phosphate } \\
\text { dehydrogenase (GAPDH) }\end{array}$ & NM_001882253 & 5'-agaacgggaagctggtcat-3' & $5^{\prime}$-ttgatgttggcgggatct-3' & 70 \\
\hline Aggrecan (ACAN) & L38480 & 5'-gaggatggcttccaccagt-3' & $5^{\prime}$-tggggtacctgacagtctga- $3^{\prime}$ & 61 \\
\hline Type I collagen chain $\alpha 1$ (Coll $\alpha 1)$ & D49399 & $5^{\prime}$-agcgatggtcctccaggt- 3 ' & 5'-gccagggtaaccacgttct- $3^{\prime}$ & 63 \\
\hline Type II collagen cha in $\alpha 1(\mathrm{Co} / 2 \alpha 1)$ & D83228 & 5'-acagcaggttcacctataccg- $3^{\prime}$ & $5^{\prime}-\operatorname{cccacttaccg} g$ tgtgtttc-3' & 60 \\
\hline
\end{tabular}

